\title{
EFEITOS DO PROGRAMA DE CAMINHADA NÓRDICA EM PORTADORAS DE FIBROMIALGIA
}

Mariele de Andrade Silva ${ }^{1}$, Maria Rita Masselli ${ }^{2}$, Sérgio Minoru Oikawa ${ }^{3}$, Raissa Puzzi Ladvig ${ }^{1}$, Thalysi Mayumi Hisamatsu ${ }^{1}$, Larissa Ruiz Teixeira ${ }^{1}$, Augusto Tavares Anheschivich ${ }^{1}$, Ana Luiza Pires Batista $^{4}$

${ }^{1}$ Graduação em Fisioterapia. ${ }^{2}$ Docente do curso de Fisioterapia. ${ }^{3}$ Docente do curso de Estatística. ${ }^{4}$ Psicóloga do CEAFIR. Departamento de Fisioterapia, Faculdade de Ciências e Tecnologia - UNESP - Presidente Prudente/SP. Email: Mariele91@hotmail.com

\section{RESUMO}

A fibromialgia é uma síndrome reumática de etiologia desconhecida e é caracterizada por dor crônica generalizada. A Caminhada Nórdica é um tipo de atividade que tem a finalidade de reduzir o impacto sobre as articulações de membros inferiores durante o exercício. O objetivo do estudo foi investigar os efeitos da caminhada nórdica em portadoras de FM. Foram selecionadas 21 portadoras de fibromialgia. Para a avaliação pré e pós-intervenção, foi avaliado o número de tender points, a qualidade de vida (através do QIF), a depressão e a ansiedade (através do HAD) e a flexibilidade. As pacientes realizaram 16 sessões de caminhada nórdica. Foram realizadas as análises com base em métodos estatísticos não paramétricos. Das 14 variáveis avaliadas, houve redução da Ansiedade $(p=0.068)$ e em 11 delas houve melhora estatisticamente significativa $(p<0.05)$. A caminhada nórdica traz vários benefícios para pacientes com fibromialgia.

Palavras -chave: Fibromialgia, Exercício Físico, Caminhada, Qualidade de Vida, Depressão.

\section{INTRODUÇÃO E OBJETIVO}

A fibromialgia é definida como uma síndrome reumática de etiologia desconhecida, que acomete predominantemente mulheres com idade entre 40 e 55 anos, e é caracterizada por dor crônica generalizada. A dor sentida pelos pacientes não tem origem inflamatória e se manifesta no sistema musculoesquelético, podendo apresentar sintomas em outros aparelhos e sistemas (CARDOSO et al., 2011). Tem sido identificados também como manifestações clínicas da fibromialgia, sintomas como: fadiga e sono não reparador, rigidez matinal, sensação de aumento do volume articular, incômodo psicológico como depressão e ansiedade, e queixas cognitivas como problemas de memória e incapacidade para concentrar-se (ÀLVARES e LIMA, 2010).

O American College of Rheumatology em 1990 definiu os critérios de classificação da FM, e estes critérios são: dor generalizada em pelo menos três dos quatro quadrantes corporais nos últimos 3 meses e dor localizada à palpação em pelo menos 11 dos 18 tender points, que são locais dolorosos preestabelecidos (HAMMERLY, 2006; MARQUES, ASSUMPÇÃO, MATSUTANI, 2007).

Segundo Berger et al. (2007), os portadores da fibromialgia (FM) utilizam mais terapias analgésicas e procuram os serviços médicos e de diagnóstico com maior frequência do que a 
população normal. O controle da síndrome é baseado na terapia multidisciplinar, por meio de intervenção farmacológica (ansiolíticos, analgésicos, antidepressivos, entre outros) e nãofarmacológica, através de estratégias que visam à atenuação dos sintomas (ALENTORN-GENI et al., 2008; BÁLSAMO e SIMÃO 2005). Estes pacientes podem se beneficiar de psicoterapia e exercícios musculoesqueléticos pelo menos duas vezes por semana, como exercícios aeróbicos moderadamente intensos, alongamento e relaxamento (HEYMANN et al., 2010). A realização do exercício físico tem sido associada também à sensação de bem-estar e à diminuição da ansiedade (VOLPATO et al., 2008). A Caminhada Nórdica (Nórdic Walking - NW) é um tipo de caminhada na qual o indivíduo caminha com auxílio de dois bastões. Esses bastões apresentam tamanhos específicos para cada indivíduo sendo recomendado que estes vão desde o chão até a altura da crista-íliaca. Através deste tipo de caminhada, os músculos dos membros superiores e músculos abdominais serão ativados e os números de passos por minuto também aumentarão levando a uma marcha mais rápida em comparação a caminhada tradicional. O resultado é um treino de corpo inteiro, que significa exercitar a condição física, solicitar mais de noventa por cento dos músculos do corpo, consumir até mais de quarenta e seis por cento de calorias, melhorar a postura e marcha e reduzir o impacto sobre as articulações durante o exercício ( $\mathrm{CHURCH}$, EARNEST, MORSS, 2002).

Um estudo investigou se um programa de 15 semanas de caminhada nórdica poderia ser uma alternativa viável em pacientes com FM e se poderia melhorar a capacidade funcional e dor nesses pacientes. Este programa foi conduzido duas vezes por semana com duração de 20 minutos de moderada a alta intensidade do exercício. Os participantes deste estudo apresentaram melhora da capacidade funcional e diminuição do nível de limitação das atividades diárias (MANNERKORPI et al., 2010). Por causa das dores frequentes nas extremidades superiores e inferiores em pacientes com FM, a caminhada nórdica pode ser um modo viável de exercício físico para essa população.

O objetivo deste estudo é investigar os efeitos do programa de caminhada nórdica sobre a qualidade de vida, depressão, ansiedade, capacidade funcional e flexibilidade em pacientes portadores de fibromialgia.

\section{METODOLOGIA}

Para realizar o estudo foram selecionadas 15 pacientes com diagnóstico de fibromialgia segundo os critérios acima mencionados. Todas as voluntárias, na faixa etária de 30 e 55 anos, 
deveriam apresentar nível cognitivo adequado para entender as orientações dadas e os procedimentos do estudo. Estas pacientes foram aconselhadas a não modificar o nível de atividades físicas no decorrer das 16 sessões. Foram excluídas do estudo pacientes que apresentavam cardiopatias. O estudo foi aprovado pela Comissão de Ética da FCT (Parecer № 192.404) e as voluntárias assinaram o Termo de Consentimento Livre e Esclarecido.

Foi avaliado o número de tender points através da palpação digital dos mesmos. Em seguida, foi avaliada a qualidade de vida através do QIF (Questionário do Impacto da Fibromialgia na Qualidade de Vida), a depressão e ansiedade através da Escala Hospitalar de Ansiedade e Depressão (HADS), o esforço percebido por meio da Escala de Percepção de Esforço, e por fim, a flexibilidade através do teste do terceiro dedo-solo.

O QIF é um questionário de qualidade de vida específico para a fibromialgia, ele apresenta 10 domínios sendo eles e suas respectivas pontuações: Capacidade Funcional (0-30), Sentir-se bem (0-7), Faltas ao trabalho (0-7), Capacidade em realizar o serviço (0-10), Dor (0-10), Fadiga (0-10), Cansaço Matinal (0-10), Rigidez (0-10), Ansiedade (0-10) e Depressão (0-10). Todas as questões apresentam melhores resultados se estiverem mais próximas do valor zero, sendo a única exceção, a questão de número 2 , em que, quanto mais próximo do valor de zero, pior o resultado (MARQUES et al., 2006)

A Escala Hospitalar de Ansiedade e Depressão (HADS) - em inglês Hospital Anxiety and Depression Scale (HADS) apresenta 14 itens dos quais sete são voltados para a avaliação da ansiedade (HAD-A) e sete para a depressão (HAD-D). Cada um dos seus itens pode ser pontuado de zero a três pontos, compondo uma pontuação máxima de 21 pontos para cada escala. Para a avaliação da frequência da ansiedade e da depressão foram obtidas as respostas aos itens da HADS. Foram adotados os pontos de cortes apontados por Zigmond e Snaith (1983) recomendados para ambas as subescalas: HAD-ansiedade: sem ansiedade de 0 a 8 , com ansiedade $\geq 9$ e HAD-depressão: sem depressão de 0 a 8, com depressão $\geq 9$ (MARCOLINO et al., 2007). Para o teste terceiro dedo-solo o padrão de normalidade é considerado tocar o solo com o terceiro dedo.

Escala de Percepção de Esforço é uma escala numérica de 6 a 20, na qual o indivíduo aponta sua própria percepção de esforço. Ela pode ser utilizada para qualquer atividade aeróbia, sendo recomendada como uma opção prática na observação da intensidade de esforço. Os números de 6-20 são baseados na frequência cardíaca de 60-200 bpms por minuto. Sendo que o 
número 12 corresponde aproximadamente $55 \%$ e o 16 a $85 \%$ da freqüência cardíaca máxima (BORG, 1982).

Após a avaliação inicial, as pacientes realizaram 16 sessões de caminhada nórdica, sendo três sessões por semana e, posteriormente, foram reavaliadas. Cada sessão foi subdividida em: 10 minutos de alongamento prévio, 30 minutos de prática de caminhada nórdica, alternando entre leve e moderada intensidade e 10 minutos de alongamento final. O local utilizado para este programa foi uma área livre dentro das dependências da Universidade Estadual de São Paulo Júlio de Mesquita Filho, Campus de Presidente Prudente.

Antes e após a atividade aeróbia, foi aferida a pressão arterial e a frequência cardíaca para que se possa prevenir riscos e a Escala de Percepção de Esforço de Borg, com o objetivo de controlar a percepção do esforço em cada sessão no início e final da realização do programa.

Para a análise estatística foram realizadas as análises exploratórias dos dados para as diferenças entre a pré e a pós-intervenção das variáveis estudadas, por meio da Mediana (como medidas de posição) e Amplitude Interquartil (AIQ) como medidas de dispersão. Para os cálculos das medidas estatísticas e a realização dos testes de Wilcoxon das variáveis estudadas, utilizou-se o software estatístico R (versão 3.0.1) livre (www.r-project.org).

\section{RESULTADOS}

Ao total foram avaliadas 21 pacientes, sendo que 7 pacientes foram excluídas do estudo atendendo aos critérios de exclusão, 5 desistiram durante a intervenção e apenas 9 pacientes deram continuidade e finalizaram as 16 sessões de caminhada nórdica. A média de idade das pacientes foi $49,11 \pm 5,18$. A Tabela 1 apresenta os valores das medianas, das amplitudes interquartis (AIQ) e o p-valor das diferenças entre a pré-intervenção e pós-intervenção das pacientes avaliadas pelo questionário QIF, HAD, tender points e flexibilidade. Observa-se também que o teste de Wilcoxon não apresentou diferenças estatísticas ( $p>0.05)$ para as variáveis número de tender points e depressão. A variável ansiedade de HAD não foi significativa estatisticamente, porém apresentou tendência $(p \approx 0.06802)$. Nas demais variáveis avaliadas, o teste de Wilcoxon mostrou que as diferenças são significativas estatisticamente $(p<0.05)$. 
Tabela1. Estimativa dos parâmetros Mediana $\left(A I Q=Q_{3}-Q_{1}\right)$ para o Questionário QIF

\begin{tabular}{|c|c|c|}
\hline Parâmetros & Diferença & P-Value \\
\hline Tender Point & $0.0(4)$ & 0.58980 \\
\hline Flexibilidade & 8.0(14) & $0.01415^{*}$ \\
\hline Capacidade Funcional & $3.0(6)$ & $0.03552 *$ \\
\hline Sentir Bem & $-2.0(2)$ & $0.02009 *$ \\
\hline Falta ao Trabalho & $3.0(6)$ & $0.03851^{*}$ \\
\hline Capacidade em realizar o Serviço & $3.0(5)$ & $0.00855^{*}$ \\
\hline Dor & $5.0(3)$ & $0.00885^{*}$ \\
\hline Fadiga & $5.0(2)$ & $0.01207^{*}$ \\
\hline Cansaço Matinal & $2.0(2)$ & $0.02154 *$ \\
\hline Rigidez & $4.0(2)$ & $0.01368 *$ \\
\hline Ansiedade & $4.0(4)$ & $0.02897^{*}$ \\
\hline Depressão & $1.0(4)$ & 0.28380 \\
\hline Ansiedade HAD & $3.0(4)$ & $0.06802 *$ \\
\hline Depressão HAD & $1.0(3)$ & $0.05747^{*}$ \\
\hline
\end{tabular}

\section{DISCUSSÃO}

Baseando-se nos resultados deste estudo, houve melhora da capacidade funcional, dor, fadiga, cansaço matinal, rigidez, ansiedade, depressão e flexibilidade nas voluntárias. No estudo de Konrad (2005), a caminhada de leve a moderada intensidade proporcionou melhoras para a fadiga e a ansiedade, e no estudo de Sttefens (2011), após a prática de 32 sessões de caminhada houve melhora de forma significativa na qualidade do sono e os estados de humor de mulheres com FM. Matsutani et al. (2012) compararam em um estudo, os efeitos de exercícios de alongamento muscular com exercícios aeróbios na dor, número de tender points, sono, ansiedade e depressão de pacientes com FM. Após o estudo, sugeriram que os exercícios de alongamento são mais eficazes que os exercícios aeróbios na dor, número de tender points, sono e depressão. Segundo estas, os exercícios aeróbios parecem produzir um efeito mais importante na diminuição da ansiedade em comparação aos exercícios de alongamento.

Como se pode observar, pelo reduzido número de concluintes, a adesão das pacientes foi muito baixa. Por se tratar de uma patologia complexa, que provoca sintomas desagradáveis para as pacientes é conhecida a baixa adesão das mesmas.

Segundo Flegal et al. (2007), a desistência da prática de exercícios físicos está vinculada à "perturbações do sono", "fadiga", "dor" e "depressão severa”, dentre outros fatores psicológicos. No estudo realizado por Schmitz et al. (2008), a dor influenciou na desistência dos praticantes de exercícios físicos. Dependendo da intensidade que é realizado o exercício aeróbio, pode ser que aumente a sensibilidade a dor. Van Sanden et al. 2002, chegou a conclusão que exercícios 
aeróbios de alta intensidade exacerbou os sintomas de portadoras de FM. Outros fatores que podem levar a baixa adesão a um programa de atividade física são as atividades ocupacionais, pois no estudo de Pinheiro et al. (2010) a maior barreira para a adesão à prática de exercícios físicos foi a jornada de trabalho excessiva e compromissos familiares.

Steffens et al. 2011, em seu estudo, relatou que a prática da caminhada envolve baixo custo e a literatura tem apresentado suas qualidades. Acredita-se que a caminhada nórdica pode ser um modo de exercitar-se mais aconselhável as portadoras de fibromialgia, pois os bastões proporcionam um apoio e equilíbrio como também baixo impacto sobre as articulações de membros inferiores. Além disso, é viável a estas pacientes, pois podem realizá-los sozinhas, ou seja, não precisam freqüentar grandes centros para a prática da atividade física.

Há necessidade de novas investigações sobre os efeitos da caminhada nórdica sobre os sinais e sintomas da fibromialgia, e estudos diversificados em termos de frequência, intensidade, tempo e tipo de exercício, pois contribuem para discussão de tratamentos que envolvam a relação custo-benefício positivo para pacientes portadoras de fibromialgia.

\section{CONCLUSÕES}

Conforme os resultados obtidos, conclui-se que as voluntárias desta pesquisa alcançaram melhoras significativas estatisticamente em 11 variáveis decorrente da prática de caminhada nórdica. Sendo assim, de acordo com os resultados alcançados e de outros estudos similares, verifica-se que essa prática de atividade física favorece as portadoras de fibromialgia.

\section{REFERÊNCIAS}

Alentorn-Geni E, Padilla J, Moras G, Haro CL, Fernández-Sola J. Six weeks of whole-body vibration exercise improves pain and fatigue in women with fibromyalgia. The Journal of Alternative and Complementary Medicine 2008;14:975-981. http://dx.doi.org/10.1089/acm.2008.0050

Álvares TT, Lima MEA. Fibromialgia - interfaces com as LER/DORT e considerações sobre sua etiologia ocupacional. Ciência \& Saúde Coletiva 2010; 15(3): 803-812. http://dx.doi.org/10.1590/S1413-81232010000300023

Bálsamo S, Simão R. Treinamento de força para Osteoporose, Fibromialgia, Diabetes tipo 2, Artrite Reumatoide e Envelhecimento. 2a ed. São Paulo: Phorte, 2005.

Berger A, Dukes E, Martin S, Edelsberg J, Oster G. Characteristics and healthcare costs of patients with fibromyalgia syndrome. Int J ClinPract 2007; 61(9):1498-508. http://dx.doi.org/10.1111/i.1742-1241.2007.01480.x 
Borg GA. Psychophysical bases of perceived exertion. Med. Sci. Sports. Exerc 1982; 14:377-381. http://dx.doi.org/10.1249/00005768-198205000-00012

Cardoso FS, Curtolo M, Natour J, Lombardi Jr I. Avaliação da qualidade de vida, força muscular e capacidade funcional em mulheres com fibromialgia. Rev. Bras. Reumatol 2011; 51(4):335-50. http://dx.doi.org/10.1590/S0482-50042011000400006

Church TS, Earnest CP, Morss GM. Field testing of Physiological Responses Associated with Nordic Walking. Research Quarterly for Exercise and Sports 2002;73(3): 296-300. http://dx.doi.org/10.1080/02701367.2002.10609023

Flegal KE, Kishiyama S, Zajdel D, et al. Adherence to yoga and exercise inter-ventions in a 6 month clinical trial. BMC Complement Altern Med (online) 2007; (7):37. http://dx.doi.org/10.1186/1472$\underline{6882-7-37}$

Hammerly M. Fibromialgia: uma nova abordagem integrativa sobre como combinar o melhor das terapias tradicional e alternativa. São Paulo: Gaia, 2006.

Heymann RE, Paiva ES, Helfenstein Jr M, Pollak DF, Martinez JE, Provenza JR, Paula AP, Althoff AC, Souza EJR, Neubarth F, Lage LV, Rezende MC, Assis MR, Lopes MLL, Jennings F, Araújo RLCC, Cristo VV, Costa EDG, Kaziyama HHS, Yeng LT, lamamura M, Saron TRP, Nascimento OJM, Kimura LK, Leite VM et al. Consenso brasileiro do tratamento da fibromialgia. Rev. Bras. Reumatol 2010;50(1):56-66. http://dx.doi.org/10.1590/S0482-50042010000100006

Konrad LM. Efeito agudo do exercício físico sobre a qualidade de vida de mulheres com síndrome da Fibromialgia. Rev. Bras. de Ativ Fis \& Saúde 2005;10(2).

Mannerkorpi K, Nordeman L, Cider A, Jonsson G. Does moderate-to-high intensity Nordic walking improve functional capacity and pain in fibromyalgia? A prospective randomized controlled trial. Arthritis Research \& Therapy 2010; 12:R189. http://dx.doi.org/10.1186/ar3159

Marcolino JAM, Mathias LAST, Piccinini Filho L, Guaratini AA, Suzuki FM, Alli LAC. Escala Hospitalar de Ansiedade e Depressão: Estudo da Validade de Critério e da Confiabilidade com Pacientes no Pré-Operatório. Rev Bras Anestesiol 2007; 57(1):52-56. http://dx.doi.org/10.1590/S0034$\underline{70942007000100006}$

Marques AP, Assumpção A, Matsutani LA. Fibromialgia e fisioterapia: avaliação e tratamento. 1ํd Barueru: Manole, 2007.

Marques AP, Santos AMB, Assumpção A, Matsutani LA, Lage LV, Pereira CAB. Validação da versão brasileira do Fibromyalgia Impact Questionnaire (FIQ). Rev. Bras. Reumatol 2006;46(1).

Matsutani LA, Assumpção A, Marques AP. Exercícios de alongamento muscular e aeróbico no tratamento da fibromialgia: estudo piloto. Rev Fisioter Mov. 2012 abr/jun; 25(2):411-8. http://dx.doi.org/10.1590/S0103-51502012000200019

Pinheiro KC, Augusto D, Silva S, Petroski, EL. Barreiras percebidas para a prática de musculação em adultos desistentes da modalidade. Rev Bras Ativ Fis Saúde 
$2010 ; 15: 157-62$.

Schmitz JAB, Souza JB, Fonseca ABP, Andrade A. Aderência de mulheres com Síndrome da Fibromialgia a um programa estruturado de hatha yoga. Rev Dor 2008;3(9):24. (RESUMO)

Steffens RAK, Liz CM, Viana MS, Brandt R, Oliveira LGA, Andrade A. Praticar caminhada melhora a qualidade do sono e os estados de humor em mulheres com síndrome da fibromialgia. Rev Dor. São Paulo 2011;12(4):327-31.

Volpato AMJ, Mendonça SAT, Pinho RA, Boeck CR. Contribuição do sistema opióide na melhora dos transtornos de ansiedade pelo exercício físico. Revista de Pesquisa e Extensão em Saúde 2008; $4(1)$.

van Santen M, Bolwijn P, Landewé R, Verstappen F, Bakker C, Hidding A, van Der Kemp D, Houben $\mathrm{H}$, van der Linden S: High or low intensity aerobic fitness training in fibromyalgia: does it matter? J Rheumatol 2002, 29:582-587.

Wilcoxon, F. Individual comparisons by ranking methods. Biometrics Bull. 1945, (I) 80-83. 